\title{
Volatility Transmission across Financial Markets: A Semiparametric Analysis
}

\author{
Theoplasti Kolaiti, Mwasi Mboya $₫$ and Philipp Sibbertsen * \\ Institute of Statistics, Faculty of Economics and Management, Leibniz University Hannover, D-30167 Hannover, \\ Germany; kolaiti@statistik.uni-hannover.de (T.K.); mboya@statistik.uni-hannover.de (M.M.) \\ * Correspondence: sibbertsen@statistik.uni-hannover.de; Tel.: +49-511-762-3783; Fax: +49-511-762-3923
}

Received: 9 June 2020; Accepted: 21 July 2020; Published: 24 July 2020

\begin{abstract}
This paper revisits the question whether volatilities of different markets and trading zones have a long-run equilibrium in the sense that they are fractionally cointegrated. We consider the U.S., Japanese and German stock, bond and foreign exchange markets to see whether there is fractional cointegration between the markets in one trading zone or for one market across trading zones. Also the other combinations of different markets in different trading zones are considered. Applying a purely semiparametric approach through the whole analysis shows fractional cointegration can only be found for a small minority of different cases. Investigating further we find that all volatility series show persistence breaks during the observation period which may be a reason for different findings in previous studies.
\end{abstract}

Keywords: high-frequency data; realized volatility; semiparametric estimation; fractional cointegration

JEL Classification: C14; C32; G15

\section{Introduction}

It is a well established fact that financial market volatility exhibits long-range dependence independent of the asset class be it foreign exchange rates, stocks or bonds and independent of the market be it European, Asian, or American. An intensive investigation of the fractional integration properties of different asset classes and many countries can be found among others in Wenger et al. (2018) using up to date estimators which also account for possible biases due to low frequency contaminations such as structural changes or trends. Nguyen et al. (2020) investigate the long memory behavior of stock market volatility for a huge range of countries and find that developed markets have stronger memory than developing markets. As a higher memory parameter leads to more persistence in the volatility and thus to a better predictability of the risk of a stock they interpret the memory of the volatility as a measure for the stability of the stock.

Besides fractional integration in single assets it is also of interest to see whether there are links between assets, asset classes or markets meaning that there is a common factor driving the volatilities. In an early study, Andersen et al. (2003) developed a framework for integration of high-frequency data for modeling and forecasting realized volatilities. In their empirical application, they heuristically tested for fractional cointegration between the realized logarithmic volatility series of three foreign exchange markets (U.S., Germany and Japan) and found no evidence of long-run linkages.

Clements et al. (2016) looked for foreign exchange rates, stocks and bonds in different markets and questions whether the volatilities of these assets have co-movements in the sense that they are fractionally cointegrated. They, in a first step, estimated the memory parameter of the individual assets by the R/S statistic and the GPH-estimator of Geweke and Porter-Hudak (1983). They found a nonstationary memory of the volatility in most cases. Afterwards they fit a parametric fractional 
VAR model to discover fractional cointegration between the asset classes and markets. Clements et al. (2016) found linkages in the asset classes between markets and discovered that they are fractionally cointegrated.

However, these results are in contradiction with other findings. Wenger et al. (2018) found lower memory parameters mainly in the stationary region which makes the concept of fractional cointegration questionable for these asset classes. This may be due to accounting for low frequency contaminations which lead to an upwards bias in the memory estimates. Nguyen et al. (2020) found different memory parameters in different markets. This would exclude fractional cointegration as equal memory parameters is a key assumption for fractional cointegration.

As the volatilities of an asset are commonly seen as a measure of the risk if this asset long memory in volatility describes the persistence of this risk. The higher the memory the more persistent the series is and the better are long-term forecasts. Therefore, the persistence of the volatility can be interpreted as a measure of stability of the asset. The higher the memory in the volatility the more stable is the asset. This interpretation of long memory in volatilities goes along with the findings in Nguyen et al. (2020) who find higher memory in more stable stocks. As fractional cointegration describes common equilibria between assets the finding of fractional cointegration between volatilities indicates the assets have a similar predictability of risk and thus stability. Therefore, from an economic point of view looking at fractional cointegration between different markets and asset classes is comparing the stability of these markets and asset classes.

At the same time, many studies focus on the impact of financial crises on the volatility spillover. A recent study by Karanasos et al. (2014) points out that volatility persistence and volatility spillovers in stock market returns show significant time variation. The authors find breaks in variance for all series connected to main economic events with global impact. For the cases of FTSE-DAX and NIKKEI-Hand Seng volatility spillovers are found, which are more pronounced in the latest financial crisis.

In this paper, we therefore reexamine the paper by Clements et al. (2016) believing that their results are driven by the combination of a semiparametric estimation of the memory parameters and a parametric modelling of the fractional cointegration relation. We follow a purely semiparametric approach by using Whittle-based estimators for the memory parameters and semiparametric tests for fractional cointegration, namely (Chen and Hurvich (2006); Souza et al. (2018); Wang et al. (2015)). By doing so we cannot confirm the results by Clements et al. (2016) for several reasons. First, our estimated memory parameters are stationary and thus volatilities do not have any stochastic trend. This is in line with the findings in Wenger et al. (2018). Second, a semiparametric test by Robinson and Yajima (2002) for equality of the memory parameters rejects this null in most cases and thus excludes fractional cointegration and third our semiparametric tests reject the null of fractional cointegration in most of the remaining cases.

Motivated by Karanasos et al. (2014) we extend our analysis by investigating potential breaks in persistence. We therefore apply the regression-based Lagrange Multiplier test introduced by Martins and Rodrigues (2014) to test for structural breaks in the memory of the volatility series. Our results exhibit breakpoints in all series during the global financial crisis and European debt crisis. We, then, apply rolling window regressions to gain further information of the persistence dynamics, which indicates a change of persistence over time. Therefore, we re-apply the persistence testing according to the estimated breakpoints and observe shifts in the order of integration during different periods.

This confirms previous findings in the literature. Chowdhury et al. (2016) study the news transmission between developed and emerging markets, namely equities, bonds and futures in different time zones. They corroborate strong time-varying characteristics in long-term cointegration and volatility spillover among equity markets from all three trading zones.

The rest of the paper is organized as follows. In Section 2, the fractional cointegration methodology is presented. Section 3 describes the data selection for the analysis. In Section 4 the empirical results are presented, while Section 5 concludes. 


\section{Methodology}

In general, (fractional) cointegration is an equilibrium concept where the persistence of the cointegrating residual $d_{v}$ determines the speed of adjustment towards the cointegration equilibrium $\beta^{\prime} X_{t}$, and shocks have no permanent influence on the equilibrium as long as $d_{v}<1$ holds. We therefore allow for fractional cointegration and consider a bivariate system of the form

$$
\begin{aligned}
X_{1 t} & =c_{1}+\xi_{1} Y_{t}+\Delta^{-\left(d-b_{1}\right)} u_{1 t} 1_{\{t>0\}} \\
X_{2 t} & =c_{2}+\xi_{2} Y_{t}+\Delta^{-\left(d-b_{2}\right)} u_{2 t} 1_{\{t>0\}} \\
Y_{t} & =\Delta^{-d} e_{t} 1_{\{t>0\}},
\end{aligned}
$$

where the coefficients $c_{1}, c_{2}, \xi_{1}$, and $\xi_{2}$ are finite, $0 \leq b_{1}, b_{2} \leq d, L$ is the lag-operator, the fractional differences $\Delta^{d} Y_{t}=(1-L)^{d} Y_{t}$ are defined in terms of generalized binomial coefficients such that

$$
\begin{aligned}
(1-L)^{d} & =\sum_{k=0}^{\infty}\left(\begin{array}{l}
d \\
k
\end{array}\right)(-1)^{k} L^{k}=\sum_{k=0}^{\infty} \pi_{k} L^{k}, \\
\text { with }\left(\begin{array}{l}
d \\
k
\end{array}\right) & =\frac{d(d-1)(d-2) \ldots(d-(k-1))}{k !},
\end{aligned}
$$

and $e_{t}$ and $u_{t}=\left(u_{1 t}, u_{2 t}\right)^{\prime}$ are martingale difference sequences. The memory of both $X_{1 t}$ and $X_{2 t}$ is determined by $Y_{t}$ so that they are integrated of the same order $d$, denoted by $X_{t} \sim I(d)$, where the memory parameter is restricted to $d \in(0,1]$ and $X_{t}=\left(X_{1 t}, X_{2 t}\right)^{\prime}$. Since it is assumed that $u_{1 t}=u_{2 t}=e_{t}=0$ for all $t \leq 0$, the processes under consideration are fractionally integrated of type-II. For a detailed discussion of type-I and type-II processes refer to Marinucci and Robinson (1999). The spectral density of $X_{t}$ can be approximated by

$$
f_{X}(\lambda) \sim \Lambda_{j}(d) G \overline{\Lambda_{j}(d)}, \quad \text { as } \lambda \rightarrow 0^{+},
$$

where $G$ is a real, symmetric, finite, and positive definite matrix, $\Lambda_{j}(d)=\operatorname{diag}\left(\lambda^{-d} e^{i \pi d / 2}, \lambda^{-d} e^{i \pi d / 2}\right)$ is a $2 \times 2$ diagonal matrix and $\overline{\Lambda_{j}(d)}$ is its complex conjugate transpose. The periodogram of a process $X_{t}$ is defined through the discrete Fourier transform $w_{X}\left(\lambda_{j}\right)=\frac{1}{\sqrt{2 \pi T}} \sum_{t=1}^{T} X_{t} e^{i \lambda_{j} t}$ as $I_{X}\left(\lambda_{j}\right)=$ $w_{X}\left(\lambda_{j}\right) \overline{w_{X}\left(\lambda_{j}\right)}$, with Fourier frequencies $\lambda_{j}=2 \pi j / T$ for $j=1, \ldots,\lfloor T / 2\rfloor$, where the operator $\lfloor\cdot\rfloor$ returns the integer part of its argument.

The two series $X_{1 t}$ and $X_{2 t}$ are said to be fractionally cointegrated, if there exists a linear combination

$$
\beta^{\prime} X_{t}=v_{t}
$$

so that the cointegrating residuals $v_{t}$ are fractionally integrated of order $I(d-b)$ for some $0<b \leq d$. Obviously, for the model in Equations (1)-(3), this is the case for every multiple of the vector $\left(1,-\frac{\xi_{1}}{\xi_{2}}\right)^{\prime}$ and $b=\min \left(b_{1}, b_{2}\right)$.

We restrict ourselves to a bivariate set-up as is common in the literature to avoid identification problems.

Here, we conclude that the financial markets, which are fractionally cointegrated with each other, are considered to be financially integrated. From the definition above, this is the case if there exists an equilibrium relationship between the financial series $\left(X_{1 t}\right.$ and $\left.X_{2 t}\right)$, such that the persistence of deviations from the equilibrium denoted by $v_{t}$ is reduced compared to that of the individual series. The degree of long memory $d-b$ in the cointegrating residuals then determines the persistence of deviations from the long-run equilibrium. This implies that series, which show a high degree of long memory in the cointegrating residuals are less firmly integrated than series with a low $d-b$. 


\section{Data}

We collect high frequency ( $5 \mathrm{~min}$ ) data from Thomson Reuters Tick History (TRTH) provided by SIRCA (The Securities Industry Research Centre of the Asia Pacific), covering the period from 22 July 1998 to 29 December 2016 for three financial markets. The data concern daily closing prices for foreign exchange, equity and bond markets for Japan, Europe and U.S. Following Clements et al. (2016), for all series we eliminate the days where one of the markets is closed or when we find that trading is significantly curtailed. Then, minimum realized volatility is formed according to Andersen et al. (2012), for a total of 3436 observations. Specifically, the dataset is constructed from the following series:

1. Yen-Dollar spot exchange contracts in each trading zone for the foreign exchange rate market,

2. NIKKEI (N225) for Japan, GDAX for Europe and S\&P 500(SPX) for U.S. in equity markets,

3. Japanese (JPY), German Bund (EUR) and United States (USD) Treasury note 10-year bond future contracts in bond markets.

The time zone set up follows Clements et al. (2016) suggestions for a global trading day, where the Japan trading zone is defined from 00:00 to 07:00 a.m., the European as 7:00 a.m. to 12:30 p.m. and United States as 12:30 p.m. to 9:00 p.m.

Analogously, we apply the Andersen et al. (2012) estimator.

$$
\operatorname{MinR}_{t}(\Delta) \equiv \frac{\pi}{\pi-2}\left(\frac{1}{1-\Delta}\right) \sum_{j=2}^{1 / \Delta} \min \left(\left|r_{j-1, t}\right|,\left|r_{j, t}\right|\right)^{2}
$$

The minRV estimator of integrated variance is based on high frequency return observations that rely on nearest neighbor truncation. The good jump robustness properties the estimator shares are also suitable for practical applications. Using Equation (5) to calculate each series for each trading zone, we then plot them in Figure 1.

Roughly, Figure 1 displays the existence of a similar pattern on the estimates of realized volatility in foreign exchange and equity data, across the three trading zones. However, foreign exchange volatility in the U.S appears to be more pronounced compared to other trading zones. On the contrary, the similarity pattern across trading zones is less pronounced in bond markets. In this scenario, the bond volatility in the U.S seems to experience more volatility events than any other trading zone. For comparison, Table 1 reports the summary of descriptive statistics for the minimized realized volatility multiplied by 1000 .

Table 1. Descriptive statistics for daily estimates of minimized realized volatility in three financial asset markets, across the three trading zones for the period 22 July 1998 to 29 December 2016.

\begin{tabular}{llllll}
\hline & Descriptive Statistics & Mean & St.dev & Skew. & Kurt. \\
\hline \multirow{2}{*}{ FX } & Japan & 0.0106 & 0.0275 & 19.549 & 515.91 \\
& Europe & 0.0096 & 0.0159 & 20.888 & 755.64 \\
& U.S. & 0.0169 & 0.0221 & 6.8067 & 84.374 \\
\hline \multirow{2}{*}{ Equity } & Japan & 0.1002 & 0.2174 & 11.690 & 198.77 \\
& Europe & 0.1473 & 0.2608 & 8.5342 & 118.96 \\
& U.S. & 0.0893 & 0.2477 & 18.272 & 561.07 \\
\hline \multirow{2}{*}{ Bond } & Japan & 0.0008 & 0.0033 & 26.132 & 974.36 \\
& Europe & 0.0055 & 0.0350 & 14.239 & 243.01 \\
& U.S. & 0.0068 & 0.0217 & 15.213 & 313.09 \\
\hline
\end{tabular}

In Table 1, we observe that the equity market provides the highest mean volatility, then the foreign exchange market, and lastly the bond market. The standard deviation volatility in the equity and bond 
market is highest during trading in the European countries, where the data are less reliable while Japan experiences the lowest standard deviation volatility, meaning that the data are more reliable. However, we observe quite the opposite in the foreign exchange market. The coefficients of both skewness and kurtosis could tell us, which financial asset market across trading zones is more interesting to the investors. Most of the investors prefer the most highest value among all, the reason being that investors would be willing to pay premium to markets, whose realized volatility are on the positive side. For our case, we observe that the Japanese bonds appear to be the most interesting financial asset market due to its highest values and U.S Foreign Exchange come last.
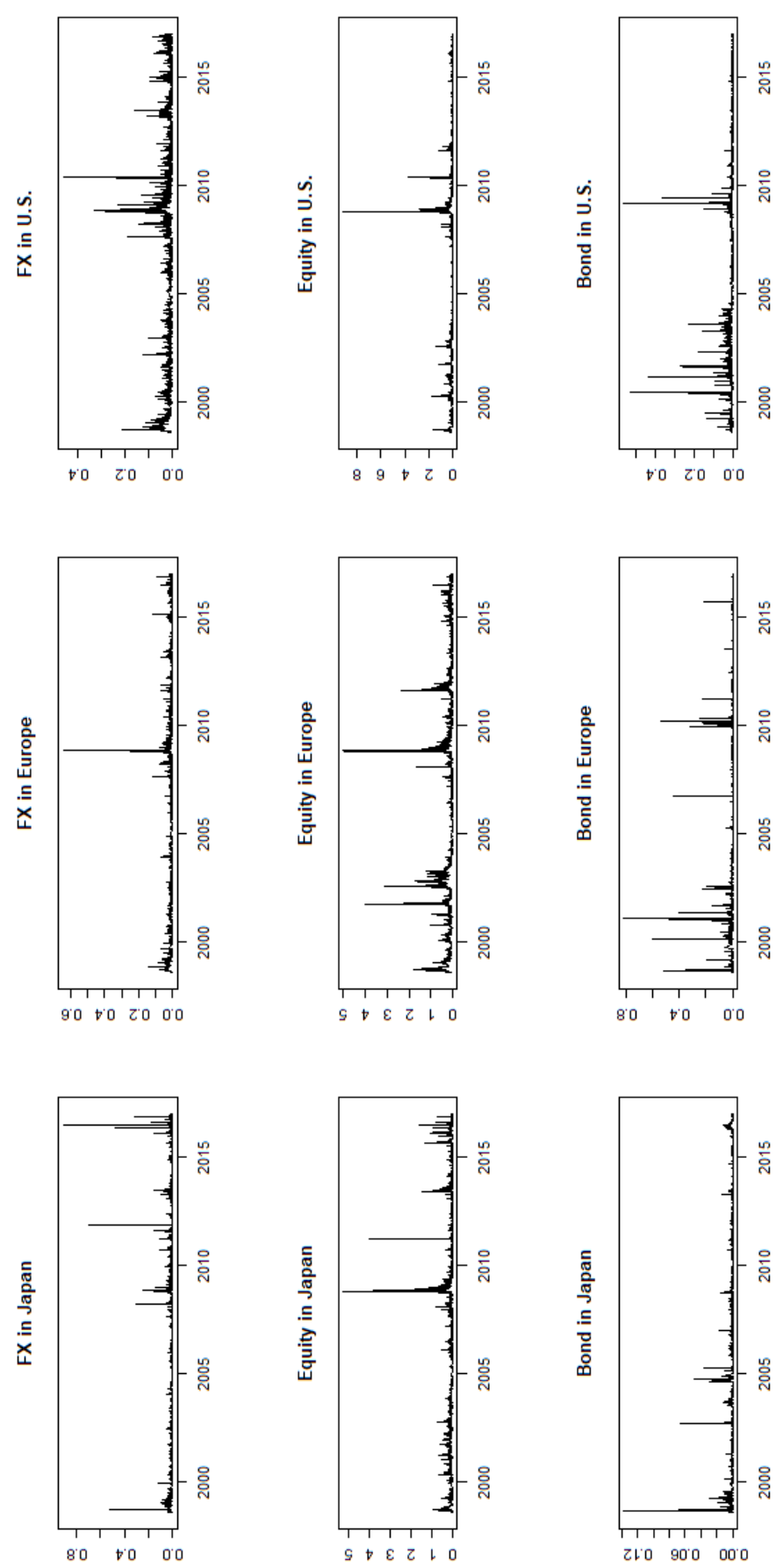

Figure 1. Minimum realized volatility estimates (scaled by 1000) for foreign exchange, equity and bond markets in Japan, Europe and U.S. 


\section{Testing for Fractional Cointegration in and across Financial Markets}

We start our analysis by estimating the order of fractional integration for each series. Memory parameters are shown in Table 2. The exact local Whittle estimator of Shimotsu (2010) is used with a $\delta=0.75$ bandwidth $m=T^{\delta}$. As a direct extension of Shimotsu and Phillips (2005), this estimator has the advantage of allowing for non-zero means, while the properties of consistency and asymptotically normal distribution for all values of $\mathrm{d}$ continue to hold.

Table 2. Memory estimates for foreign exchange, equity and bond markets.

\begin{tabular}{lccc}
\hline Financial Market & Forex & Equity & Bonds \\
\hline Japan & 0.21 & 0.40 & 0.28 \\
Europe & 0.32 & 0.44 & 0.26 \\
U.S. & 0.41 & 0.42 & 0.22 \\
\hline Note: ELW estimates, d, based on bandwidth $m=T^{0.75}$.
\end{tabular}

Table 2 suggests the existence of long memory in all series under examination. All time series are stationary and the equity market is the most persistent with memory parameters between 0.4 and 0.44 . Equity markets therefore seem to be more stable than exchange rate and bond markets for all areas.

We apply several semiparametric tests for the null hypothesis of no fractional cointegration. The advantage of semiparametric methods is that we do not impose any assumptions on the short-run behavior of the series, apart from mild regularity conditions. Thus, we can avoid spurious findings that might arise due to misspecification. Research on semiparametric tests for fractional cointegration has been an active field in recent years and there exist a variety of competing approaches. Whereas some approaches rely on the spectral representation of multivariate long memory processes and test whether the spectral matrix $G$ has full rank or not, other tests are residual-based and test for the strength of integration of the cointegration residuals. To make sure that our results are robust to the way of testing, we apply tests from both strands of the literature.

Souza et al. (2018) use the fractionally differenced process $\Delta^{d} X_{t}$ and the fact that the determinant $D_{\Delta^{d}}(\lambda)$ of $f_{\Delta^{d} X}(\lambda)$ is of the form $D_{\Delta^{d}}(\lambda) \sim \tilde{G}\left|1-e^{-i \lambda}\right|^{2 b}$, where $\tilde{G}$ is a scalar constant and $0<\tilde{G}<\infty$. An estimate of $b$ can therefore be obtained via a log-periodogram regression and the null hypothesis that $b=0$ can be tested based on the resulting estimate. We denote this spectral-based test in the following as SRFB18.

The test of Wang et al. (2015) (denoted by WWC15) is based on the sum over the fractionally differenced process $\Delta^{\hat{d}_{v}} X_{2 t}$, where $\hat{d}_{v}$ is an estimate of the memory from the cointegrating residuals obtained using a consistent estimator for the cointegrating vector $\beta$ such as the narrow-band least squares estimator of Robinson (1994), Robinson and Marinucci (2003), and Christensen and Nielsen (2006), among others. In contrast to that, the test of Chen and Hurvich (2006) (denoted by CH06) is directly based on $\hat{d}_{v}$, but the cointegrating space is estimated by the eigenvectors of the averaged and tapered periodogram matrix local to the origin. Obviously, these two tests are residual-based.

Leschinski et al. (2020) suggest that the three testing procedures have best performance among a group of eight semiparametric tests, particularly when testing for fractional cointegration. In bivariate cases, SRFB18 presents the best performance. Following their finding, we perform all tests at the 5\% significance level. The bandwidth is selected as $m=T^{0.75}$ for all three testing procedures. The trimming parameter $r$ is set to 3 for SRFB18 and the integer for averaging the periodogram is 25 for CH06.

In our analysis, we test the full sample for stationary fractional cointegration as the order of integration for all series is less than 0.5. As fractional cointegration needs as a core assumption that the order of integration is equal between the series, we use pairwise tests as suggested by Robinson and Yajima (2002) to test for the equality of the memory parameters. The results shown in Table 3 suggest that the hypothesis of a common memory parameter is rejected for most of the cases.

Given the results in Table 4, we proceed our analysis by testing for fractional cointegration under the null hypothesis of no fractional cointegration. First, we test in each of the financial markets. Specifically, we are allowed to test between the pairs, Japanese bonds (JPY)- Japanese Foreign Exchange 
(FX-JP), European Bonds (EUR)-European Foreign Exchange (FX-EU) and U.S. Foreign Exchange (FX-US) and S\&P 500 index (SPX). Table 4 suggests the existence of fractional cointegration for the Japanese and U.S. financial markets. The European market is not rejecting the null hypothesis for all three testing procedures.

Table 3. Test statistics for common order of integration.

\begin{tabular}{lccccccccc}
\hline & FX-JP & FX-EU & FX-US & N225 & GDAX & SPX & JPY & EUR & USD \\
\hline FX-JP & - & $2.6636^{* *}$ & $4.409^{* *}$ & $4.1923^{* *}$ & $4.8657^{* *}$ & $4.2236^{* *}$ & 1.3670 & 1.1788 & 0.2246 \\
FX-EU & $2.6636^{* *}$ & - & $2.0231^{* *}$ & 1.7486 & $2.5662^{* *}$ & 1.9003 & 0.9595 & 1.1377 & $2.0880^{* *}$ \\
FX-US & $4.409^{* *}$ & $2.0231^{* *}$ & - & 0.1861 & 0.6581 & 0.1355 & $2.7142^{* *}$ & $2.8837^{* *}$ & $3.8437^{* *}$ \\
N225 & $4.1923^{* *}$ & 1.7486 & 0.1861 & - & 0.8970 & 0.3414 & $2.5483^{* *}$ & $2.7031^{* *}$ & $3.6541^{* *}$ \\
GDAX & $4.8657^{* *}$ & $2.5662^{* *}$ & 0.6581 & 0.8970 & - & 0.6317 & $3.3375^{* *}$ & $3.4895^{* *}$ & $4.4417^{* *}$ \\
SPX & $4.2236^{* *}$ & 1.9003 & 0.1355 & 0.3414 & 0.6317 & - & $2.8357^{* *}$ & $2.9934^{* *}$ & $3.9513^{* *}$ \\
JPY & 1.3670 & 0.9595 & $2.7142^{* *}$ & $2.5483^{* *}$ & $3.3375^{* *}$ & $2.8357^{* *}$ & - & 0.1821 & 1.1313 \\
EUR & 1.1788 & $1.1377^{* *}$ & $2.8837^{* *}$ & $2.7031^{* *}$ & $3.4895^{* *}$ & $2.9934^{* *}$ & 0.1821 & - & 0.9528 \\
USD & 0.2246 & $2.0880^{* *}$ & $3.8437^{* *}$ & $3.6541^{* *}$ & $4.4417^{* *}$ & $3.9513^{* *}$ & 1.1313 & 0.9528 & - \\
\hline
\end{tabular}

Note: The critical value at $\alpha=5 \%$ is 1.9599 . ${ }^{* *}$ denotes significance of the test statistic at the $5 \%$ level.

Table 4. Test statistics for stationary fractional cointegration in each financial market.

\begin{tabular}{llll}
\hline Financial Markets & CH06 & SRFB18 & WWC15 \\
\hline JPY/FX-JP & $2.6342^{* *}$ & 0.1949 & $4.6768^{* *}$ \\
EUR/FX-EU & -0.9419 & -0.1789 & 0.7119 \\
FX-US/SPX & $1.9877^{* *}$ & $2.0523^{* *}$ & 1.7187 \\
\hline
\end{tabular}

Note: Critical values at $\alpha=5 \%$ are 1.9600 for both SRFB18 and WWC15, as well as 1.3860 for CH06. ${ }^{* *}$ denotes significance of the test statistic at the $5 \%$ level.

Next we investigate the cointegrating relationships across the three trading zones by testing all possible cases. We take pairwise combinations for all series and present them in three sections. First, we consider the markets of Japan and Europe.

In Table 5, cointegration exists between European Foreign Exchange and the Nikkei index, as well as for the pair of equities, N225 and GDAX. For the case of Japanese bonds and European Foreign Exchange, evidence of cointegration is only suggested by WWC15. No fractional cointegration is found for the case of the Japanese Foreign Exchange/European bonds and the pairwise case of bonds' market.

Table 5. Test statistics for stationary fractional cointegration between Japan and Europe.

\begin{tabular}{llll}
\hline Japan/Europe & CH06 & SRFB18 & WWC15 \\
\hline FX-JP/EUR & 1.3338 & -1.1062 & 1.9009 \\
N225/FX-EU & $5.4214^{* *}$ & $2.3573^{* *}$ & $6.0193^{* *}$ \\
N225/GDAX & $1.5729^{* *}$ & 0.2280 & $2.3208^{* *}$ \\
JPY/FX-EU & -0.4471 & 0.6307 & $5.0654^{* *}$ \\
JPY/EUR & -0.0509 & 0.0496 & 1.0743 \\
\hline
\end{tabular}

Note: Critical values at $\alpha=5 \%$ are 1.9600 for both SRFB18 and WWC15, as well as 1.3860 for CH06. ${ }^{* *}$ denotes significance of the test statistic at the $5 \%$ level.

We continue then testing between European and U.S. market in Table 6. Two testing procedures suggest cointegration for the pair of equities. The Chen and Hurvich (2006) testing procedure additionally rejects for the cases between the bond markets and among the U.S index and European Foreign Exchange markets. 
Table 6. Test statistics for stationary fractional cointegration between Europe and U.S.

\begin{tabular}{llll}
\hline Europe/U.S. & CH06 & SRFB18 & WWC15 \\
\hline FX-US/GDAX & 1.2347 & -0.1114 & 1.9152 \\
SPX/FX-EU & $3.1747^{* *}$ & 0.2334 & 1.9598 \\
SPX/GDAX & $1.8900^{* *}$ & -0.0929 & $2.5757^{* *}$ \\
USD/EUR & $2.2552^{* *}$ & 0.4272 & 1.8357 \\
\hline
\end{tabular}

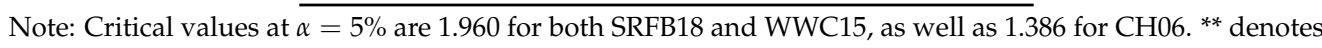
significance of the test statistic at the $5 \%$ level.

Last, in Table 7, U.S-Japanese markets show evidence of cointegration for all four pairs. Equity markets and FX-U.S./N225 pairs are rejecting in all three testing procedures. Both SRFB18 and WWC15, reject the pair of U.S. bonds and Japanese Foreign Exchange. The Bond's market pair is rejected only by the WWC15 test.

Table 7. Test statistics for stationary fractional cointegration between U.S. and Japan.

\begin{tabular}{llll}
\hline U.S./Japan & CH06 & SRFB18 & WWC15 \\
\hline FX-US/N225 & $3.6269^{* *}$ & $3.5741^{* *}$ & $3.6080^{* *}$ \\
SPX/N225 & $4.3794^{* *}$ & $4.9149^{* *}$ & $4.1686^{* *}$ \\
USD/FX-JP & 0.2946 & $2.4754^{* *}$ & $4.8512^{* *}$ \\
USD/JPY & -1.1685 & 1.7754 & $3.5412 * *$ \\
\hline
\end{tabular}

Note: Critical values at $\alpha=5 \%$ are 1.960 for both SRFB18 and WWC15, as well as 1.386 for CH06. ${ }^{* *}$ denotes significance of the test statistic at the $5 \%$ level.

As a next step, we estimate the cointegration vector $\beta$ as well as the memory parameters of the residuals $d_{v}$ by using Narrow Band Least Squares estimation. Robinson (1994) shows that NBLS estimation is consistent under stationary cointegration whereas an OLS approach might not retain consistency. Table 8 displays the estimated values of the memory of the residuals. We include the pairs for which the hypothesis of a common memory parameter is not rejected and at least one of the testing procedures for no cointegration rejects. Results in bold indicate the cases where the memory of $d_{v}$ is less than both of the individuals series. Here, the likelihood of fractional cointegration is fulfilled in the cases of all three equity markets, as well as between U.S bonds and European bonds.

Table 8. Memory estimates on the residuals.

\begin{tabular}{lccccccccc}
\hline & FX-JP & FX-EU & FX-US & N225 & GDAX & SPX & JPY & EUR & USD \\
\hline FX-JP & & & & & & & 0.28 & 0.22 \\
FX-EU & & & & 0.40 & & 0.42 & 0.28 & \\
FX-US & & & & 0.40 & & 0.41 & & & \\
N225 & & 0.40 & 0.40 & & $\mathbf{0 . 3 7}$ & $\mathbf{0 . 3 0}$ & & & \\
GDAX & & 0.42 & 0.41 & $\mathbf{0 . 3 0}$ & $\mathbf{0 . 3 5}$ & & & & \\
SPX & & & & $\mathbf{0 . 3 7}$ & & $\mathbf{0 . 3 5}$ & & \\
JPY & 0.28 & 0.28 & & & & & & & 0.22 \\
EUR & & & & & & & 0.22 & $\mathbf{0 . 2 0}$ & \\
USD & 0.22 & & & & & & 0.20 \\
Note: ELW estimates, $d_{v}$, based on bandwidth $m=T^{0.75}$. Bold numbers indicate the cases where $d_{v}<$
\end{tabular}

In addition to our analysis of fractional cointegration, we investigate further the existence of breaks in persistence. Hence, we perform a regression-based Lagrange Multiplier Test introduced by Martins and Rodrigues (2014) that generalizes the conventional integration approaches to the fractional integrated process context. In what follows we will denote by $\hat{\tau}$ the estimated point of the persistence shift and by $d_{1}$ the order of integration before the shift and by $d_{2}$ the order of integration after the break. Table 9 suggests evidence of a break in volatility persistence for all financial series. The overwhelming majority suggests a decrease in persistence during the global financial crisis. For four out of nine financial series, the memory reduction shift occurs in October, 2008 which are also the earliest memory 
shifts while the latest take place during the second trimester of 2013. Additionally, we find evidence for an increase in the memory parameter for the Japanese equity and bonds' market in October, 2008 and April, 2013 respectively. Comparing $d_{1}$ and $d_{2}$ the majority of the shifts is within the stationary region $0<d \leq 0.5$, suggesting lower levels of volatility persistence following the breakpoints. The stronger shift in terms of fractional integration is found for Japanese bonds which jump from 0.23 to 0.67 suggesting mean reverting non-stationarity for the second sub-sample. In general, equity markets appear to be more persistent and marginally non-stationary before the estimated memory shifts.

Table 9. Persistence change test.

\begin{tabular}{lcccc}
\hline & Test-Statistic & Date & $\boldsymbol{d}_{\mathbf{1}}$ & $\boldsymbol{d}_{\mathbf{2}}$ \\
\hline FX-JP & $-4.8797^{* * *}$ & $31 / 10 / 2008$ & 0.44 & 0.10 \\
FX-EU & $-11.5116^{* * *}$ & $24 / 10 / 2008$ & 0.40 & 0.27 \\
FX-US & $-7.4773^{* * *}$ & $16 / 01 / 2009$ & 0.47 & 0.33 \\
N225 & $-3.9495^{* * *}$ & $06 / 10 / 2008$ & 0.42 & 0.44 \\
& $-7.5297^{* * *}$ & $07 / 06 / 2013$ & 0.43 & 0.27 \\
GDAX & $-2.6691^{*}$ & $23 / 10 / 2008$ & 0.54 & 0.37 \\
SPX & $-10.7839^{* * *}$ & $10 / 10 / 2008$ & 0.59 & 0.38 \\
JPY & $-4.5658^{* * *}$ & $05 / 04 / 2013$ & 0.23 & 0.67 \\
EUR & $-11.4874^{* * *}$ & $05 / 03 / 2010$ & 0.29 & 0.06 \\
USD & $-9.1499^{* * *}$ & $12 / 02 / 2009$ & 0.22 & 0.13 \\
\hline
\end{tabular}

Note: ${ }^{* *}$ and $*$ indicate levels of significance at $1 \%$ and $10 \%$, respectively. " $d_{1}$ " and " $d_{2}$ " refer to the memory parameters of the respective subperiod of the estimated breakpoint.

Taking into account the memory reduction for most cases in October, 2008, we test for fractional cointegration during the corresponding subperiods. The results are illustrated in Tables 10 and 11 , respectively. For the period before the beginning of the financial crisis we find more cointegrating relationships, compared to the full sample period and the second subperiod, that do not reject the common memory hypothesis. However, five pairs do reject the null hypothesis of fractional cointegration and exhibit a memory of residuals less than the individual series. Here, markets appear to return to equilibrium with relative persistence, except in the case of the Japanese/European foreign exchange. For this case, the residuals are close to 0 . On contrary, the subperiod concerning the financial crisis rejects for most cases the common memory hypothesis. Finally, cointegration is found only between the U.S. and Europe, for the cases of foreign exchange, as well as for their equity markets.

Table 10. Results for stationary fractional cointegration for the subsample 22/07/1998-31/10/2008.

\begin{tabular}{llllllll}
\hline Financial Markets & $\boldsymbol{d}_{\boldsymbol{A}}$ & $\boldsymbol{d}_{\boldsymbol{B}}$ & RY02 & CH06 & SRFB18 & WWC15 & $\boldsymbol{d}_{\boldsymbol{u}}$ \\
\hline FX-JP/FX-EU & 0.32 & 0.32 & 0.0507 & $7.8396^{* *}$ & $10.5175^{* *}$ & $17.0289^{* *}$ & 0.09 \\
N225/GDAX & 0.42 & 0.47 & 1.0529 & $1.8603^{* *}$ & 1.7827 & $2.6977^{* *}$ & 0.31 \\
SPX/GDAX & 0.46 & 0.42 & 0.7889 & $3.0535^{* *}$ & 1.7315 & 4.3726 & 0.28 \\
FX-US/FX-JP & 0.43 & 0.32 & 1.8974 & $6.1552^{* *}$ & $9.4360^{* *}$ & $7.5481^{* *}$ & 0.29 \\
SPX/N225 & 0.46 & 0.47 & 0.3601 & $4.9964^{* *}$ & $2.8367^{* *}$ & $8.8679^{* *}$ & 0.30 \\
\hline
\end{tabular}

Note: $d_{A}, d_{B}$ refer to the memory estimators of the individuals series on the first column. RY02 refers to Robinson and Yajima (2002) test for common memory parameters. Critical values at $\alpha=5 \%$ are 1.960 for RY02, SRFB18 and WWC15, as well as 1.386 for CH06. ${ }^{* *}$ denotes significance of the test statistic at the $5 \%$ level. Last, $d_{u}$ is the memory of the estimated residuals.

In order to gain further insights into the dynamics of volatility persistence among the financial series and the respective trading zones, we apply a rolling window estimation. Our rolling window approach estimates the memory parameter $d$ by using a window size set of 250 observations, which corresponds to one trading year. As before, we use the ELW estimator of Shimotsu (2010) based on the bandwidth parameter of $\delta=0.75$. 
Table 11. Results for stationary fractional cointegration for the subsample 04/11/2008-29/12/2016.

\begin{tabular}{llllllll}
\hline Financial Markets & $\boldsymbol{d}_{\boldsymbol{A}}$ & $\boldsymbol{d}_{\boldsymbol{B}}$ & RY02 & CH06 & SRFB18 & WWC15 & $\boldsymbol{d}_{\boldsymbol{u}}$ \\
\hline FX-US/FX-EU & 0.39 & 0.37 & 0.2788 & $5.8332^{* *}$ & $3.6597^{* *}$ & $15.9112^{* *}$ & 0.36 \\
SPX/GDAX & 0.51 & 0.55 & 0.6660 & $4.5094^{* *}$ & $4.8607^{* *}$ & $4.1734^{* *}$ & 0.35
\end{tabular}

Note: $d_{A}, d_{B}$ refer to the memory estimators of the individuals series on the first column. RY02 refers to Robinson and Yajima (2002) test for common memory parameters. Critical values at $\alpha=5 \%$ are 1.960 for RY02, SRFB18 and WWC15, as well as 1.386 for CH06. ** denotes significance of the test statistic at the 5\% level. Last, $d_{u}$ is the memory of the estimated residuals.

Figure 2 presents the results of the rolling window fractional integration estimations for each of the nine financial series along with their $95 \%$ confidence bands. The bold vertical line corresponds to the respective estimated breakpoint. For the European and U.S. markets, we observe a downward trend after the corresponding breaks in persistence which shifts upwards again around 2013. This fluctuation may reflect the duration of the global financial crisis. Japanese equity market exhibit shifts of persistence in both directions. A marginal increase in October, 2008 followed by minor declines which finally decays after the break in June, 2013. Foreign exchange follows a similar pattern, decreasing in October, 2008, increasing in 2013 and finally remains in lower levels of integration. Japanese bonds do not exhibit significant shifts until 2013 and then show levels of higher persistence in the end of the sample. Here, a profound change in persistence does not always synchronize with the indicated breakpoint line. However, we consider that change/movement within a one year band coincides with the estimated breakpoint.

To conclude our analysis, we re-apply the rolling window estimation to the subsamples indicated by the breaks. We find a second breakpoint for all series in the first subsample and for the majority during the second subperiod. We find evidence of memory parameters in the lower stationary region in bond markets before 2005 and shifts from the high stationary to the low non-stationary range and vice versa for the rest of the cases. Results are shown in Table 12.

Table 12. Persistence change test in subsamples.

\begin{tabular}{lccccc}
\hline & \multicolumn{1}{c}{ Subsample } & Test-Statistic & Date & $\boldsymbol{d}_{\mathbf{1}}$ & $\boldsymbol{d}_{\mathbf{2}}$ \\
\hline FX-JP & $22 / 07 / 1998: 31 / 10 / 2008$ & $-6.8524^{* * *}$ & 14 June 2007 & 0.33 & 0.69 \\
FX-EU & $22 / 07 / 1998: 24 / 10 / 2008$ & $-3.1452^{* *}$ & 19 March 2007 & 0.39 & 0.05 \\
& $27 / 10 / 2008: 29 / 12 / 2016$ & $-7.2991^{* * *}$ & 21 April 2015 & 0.43 & 0.28 \\
FX-US & $22 / 07 / 1998: 16 / 01 / 2009$ & $-3.8354^{* * *}$ & 3 April 2007 & 0.41 & 0.52 \\
& $20 / 01 / 2009: 29 / 12 / 2016$ & $-3.9788^{* * *}$ & 6 February 2013 & 0.29 & 0.44 \\
N225 & $07 / 10 / 2008: 07 / 06 / 2013$ & $-10.4550^{* * *}$ & 16 March 2011 & 0.65 & 0.20 \\
& $10 / 06 / 2013: 29 / 12 / 2016$ & $-2.1367^{*}$ & 17 February 2016 & 0.27 & 0.16 \\
GDAX & $22 / 07 / 1998: 23 / 10 / 2008$ & $-2.7557^{* *}$ & 16 July 2002 & 0.34 & 0.61 \\
SPX & $22 / 07 / 1998: 10 / 10 / 2008$ & $-5.6951^{* * *}$ & 10 July 2002 & 0.4 & 0.14 \\
JPY & $22 / 07 / 1998: 05 / 04 / 2013$ & $-3.0646^{* * *}$ & 11 August 2004 & 0.15 & 0.28 \\
& $08 / 04 / 2013: 29 / 12 / 2016$ & $-15.5028^{* * *}$ & 12 April 2016 & 0.38 & 0.78 \\
EUR & $22 / 07 / 1998: 05 / 03 / 2010$ & $-3.9225^{* * *}$ & 28 May 2002 & 0.28 & -0.05 \\
USD & $22 / 07 / 1998: 12 / 02 / 2009$ & $-3.7465^{* * *}$ & 8 September 2001 & 0.06 & 0.10 \\
& $13 / 02 / 2009: 29 / 12 / 2016$ & $-2.2775^{* *}$ & 14 May 2015 & 0.20 & 0.33 \\
\hline
\end{tabular}

Note: ${ }^{* *}, * *$ and ${ }^{*}$ indicate levels of significance at $1 \%, 5 \%$ and $10 \%$, respectively. " $d_{1}$ " and " $d_{2}$ " refer to the memory parameters of the respective subperiod of the estimated breakpoint. 

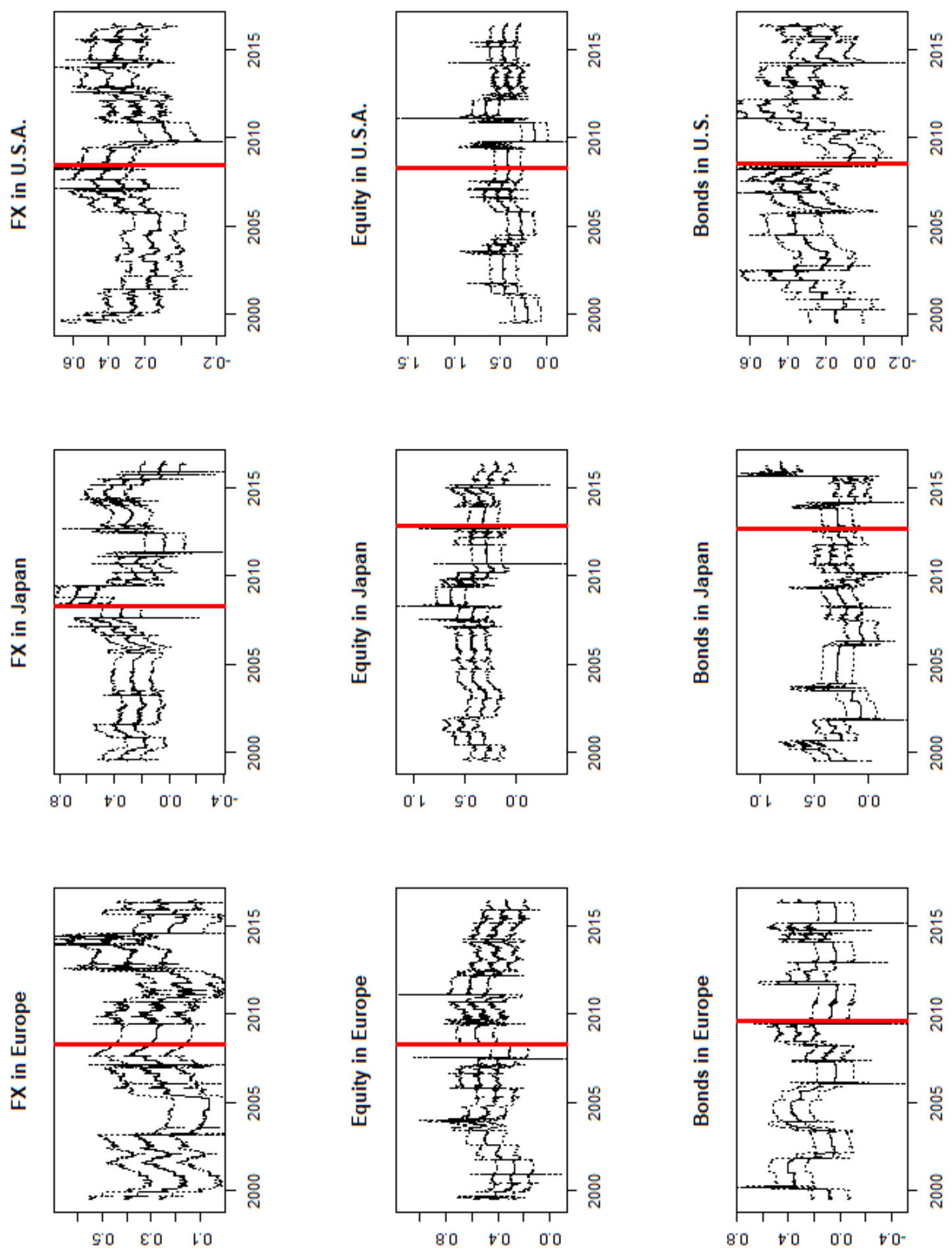

Figure 2. Rolling window estimates for foreign exchange, equity and bond markets in Japan, Europe and U.S. Bold vertical line corresponds to the estimated breakpoint.

\section{Conclusions}

In this paper, we were interested to empirically examine the volatility transmission between the major financial markets. We applied recent methods of semiparametric analysis of fractional integration and cointegration. We found that the fractional integration parameter of all series is in 
the stationary region so that strong fractional cointegration is already excluded. Furthermore, most of the series have different memory parameters once again excluding fractional cointegration. For the remaining series we found fractional cointegration among equity markets and U.S.-EU bonds. Our results are in contrast with Clements et al. (2016) who found fractional cointegration between the different markets and trading zones. The main difference in our approach is that Clements et al. (2016) used a parametric VAR model without a priori testing for the equality of the memory parameters. From an economic perspective we thus found that the risk behavior and stability of the different markets and areas is clearly distinct. This allows investors for risk diversification by investing in different asset classes and markets which is along with the intuition.

A possible reason for the different results are breaks in persistence around global financial crisis. We find a lower volatility persistence after the breaks. This can result in spurious results on fractional cointegration if neglected in the analysis. We therefore commit to taking into account the frequent changes in persistence over time.

Author Contributions: T.K.: General idea of the paper, data collection, conducting empirical analysis, upwriting of the paper; M.M.: General idea of the paper, conducting empirical analysis, upwriting of the paper; P.S.: General idea of the paper, upwriting of the paper. All authors have read and agreed to the published version of the manuscript.

Funding: This research received no external funding.

Conflicts of Interest: The authors declare no conflict of interest.

\section{References}

Andersen, Torben G., Tim Bollerslev, Francis X. Diebold, and Paul Labys. 2003. Modeling and forecasting realized volatility. Econometrica 71: 579-625. [CrossRef]

Andersen, Torben G., Dobrislav Dobrev, and Ernst Schaumburg. 2012. Jump-robust volatility estimation using nearest neighbor truncation. Journal of Econometrics 169: 75-93. [CrossRef]

Chen, Willa W., and Clifford M. Hurvich. 2006. Semiparametric estimation of fractional cointegrating subspaces. The Annals of Statistics 34: 2939-79. [CrossRef]

Chowdhury, Anup, Moshfique Uddin, and Keith Anderson. 2016. Volatility Spillovers and Time-Zone Effect: New Evidence from Emerging Markets across three Different Time Zones. Working Paper. Available online: https:/ / pdfs.semanticscholar.org/d68c/3d012993f1800416981a0a891837c8384530.pdf (accessed on 23 July 2020).

Christensen, Bent Jesper, and Morten Ørregaard Nielsen. 2006. Asymptotic normality of narrow-band least squares in the stationary fractional cointegration model and volatility forecasting. Journal of Econometrics 133: 343-71. [CrossRef]

Clements, Adam, A.S. Hurn, and Vladimir Vladimirovich Volkov. 2016. Common trends in global volatility. Journal of International Money and Finance 67: 194-214. [CrossRef]

Geweke, John, and Susan Porter-Hudak. 1983. The estimation and application of long memory time series models. Journal of Time Series Analysis 4: 221-38. [CrossRef]

Karanasos, Menelaos, Alexandros G. Paraskevopoulos, Faek Menla Ali, Michail Karoglou, and Stavroula Yfanti. 2014. Modelling stock volatilities during financial crises: A time varying coefficient approach. Journal of Empirical Finance 29: 113-28. [CrossRef]

Leschinski, Christian, Michelle Voges, and Philipp Sibbertsen. 2020. A comparison of semiparametric tests for fractional cointegration. Statistical Papers, forthcoming. [CrossRef]

Marinucci, Domenico, and Peter M. Robinson. 1999. Alternative forms of fractional Brownian motion. Journal of Statistical Planning and Inference 80: 111-22. [CrossRef]

Martins, Luis F., and Paulo M. M. Rodrigues. 2014. Testing for persistence change in fractionally integrated models: An application to world inflation rates. Computational Statistics \& Data Analysis 76: 502-22.

Nguyen, Duc Binh Benno, Marcel Prokopczuk, and Philipp Sibbertsen. 2020. The memory of stock return volatility: Asset pricing implications. Journal of Financial Markets 47: 100487. [CrossRef]

Robinson, Peter M., and D. Marinucci. 2003. Semiparametric Frequency Domain Analysis of Fractional Cointegration. London: London School of Economics and Political Science, LSE Library. 
Robinson, Peter M. 1994. Semiparametric analysis of long-memory time series. The Annals of Statistics 1: 515-39. [CrossRef]

Robinson, Peter M., and Yoshihiro Yajima. 2002. Determination of cointegrating rank in fractional systems. Journal of Econometrics 106: 217-41. [CrossRef]

Shimotsu, Katsumi. 2010. Exact local whittle estimation of fractional integration with unknown mean and time trend. Econometric Theory 26: 501-40. [CrossRef]

Shimotsu, Katsumi, and Peter C. B. Phillips. 2005. Exact local whittle estimation of fractional integration. The Annals of statistics 33: 1890-933. [CrossRef]

Souza, Igor Viveiros Melo, Valderio Anselmo Reisen, Glaura da Conceição Franco, and Pascal Bondon. 2018. The estimation and testing of the cointegration order based on the frequency domain. Journal of Business $\mathcal{E}$ Economic Statistics 36: 695-704.

Wang, Bin, Man Wang, and Ngai Hang Chan. 2015. Residual-based test for fractional cointegration. Economics Letters 126: 43-46. [CrossRef]

Kai R. Wenger, Christian H. Leschinski, Philipp Sibbertsen. 2018. The Memory of Volatility. Quantitative Finance and Economics 2: 137-59. [CrossRef]

(C) 2020 by the authors. Licensee MDPI, Basel, Switzerland. This article is an open access article distributed under the terms and conditions of the Creative Commons Attribution (CC BY) license (http:/ / creativecommons.org/licenses/by/4.0/). 